LOS ENTORNOS VIRTUALES DE APRENDIZAJE: UN NUEVO CAMINO HACIA EL CONOCIMIENTO

Maiby T. Camacho G y Marcos E. Flores G

Pp 96-109

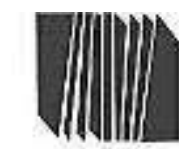

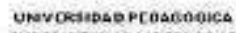

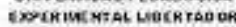

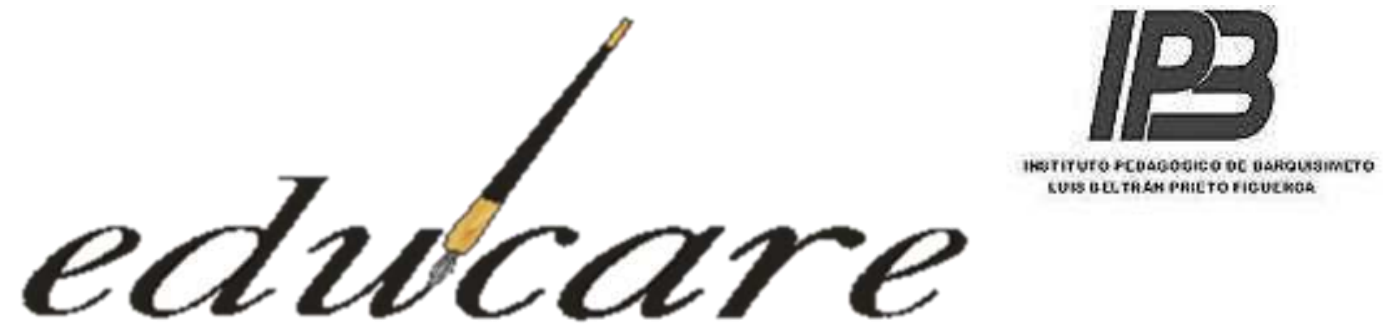

Órgano Divulgativo de la Subdirección de Investigación y Postgrado del Instituto Pedagógico de Barquisimeto "Lıis Beltrán Prieto

Figueroa"

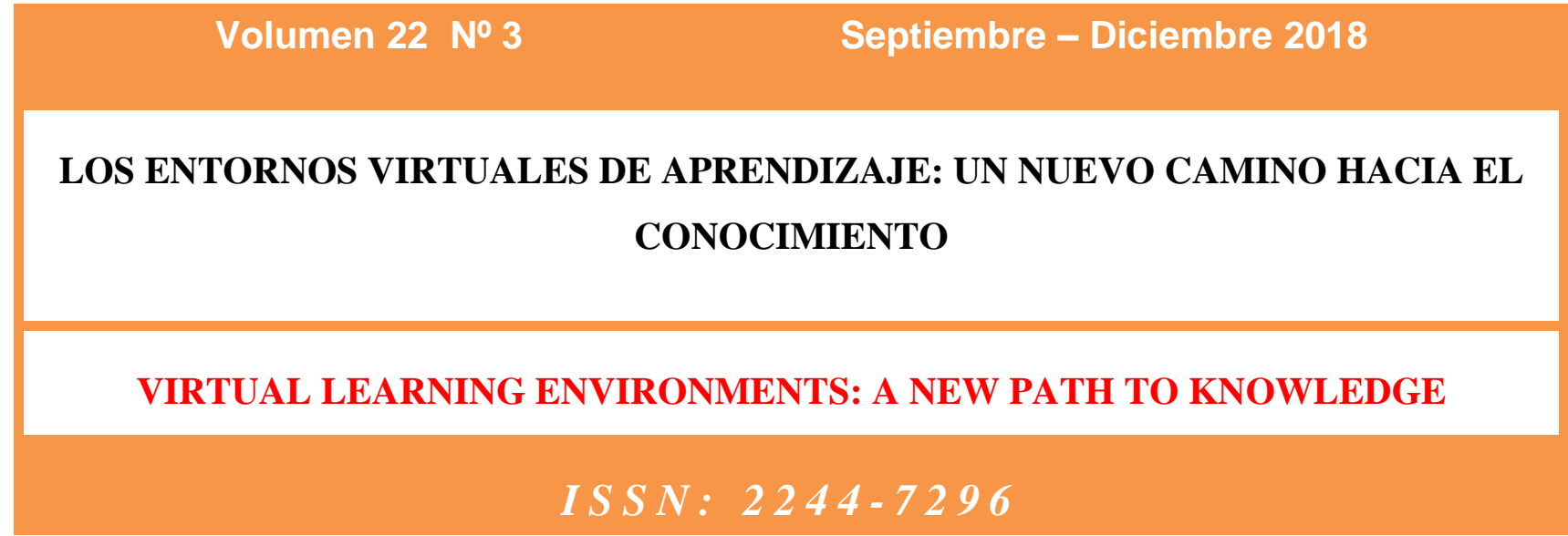

\author{
Autor \\ Maiby Camacho G \\ Universidad Centroccidental "Lisandro Alvarado" (UCLA) \\ Marcos Flores G \\ Universidad Pedagógica Experimental Libertador \\ Instituto Pedagógico de Barquisimeto (UPEL-IPB) \\ Venezuela
}




\section{LOS ENTORNOS VIRTUALES DE APRENDIZAJE: UN NUEVO CAMINO HACIA EL CONOCIMIENTO}

\section{Maiby T. Camacho G y Marcos E. Flores G}

Pp 96-109

Ensayo

Recibido:15-02-2018

\section{RESUMEN}

En el mundo globalizado las tecnologías posibilitan nuevos tipos de aprendizajes y de difusión del conocimiento surgiendo diversos escenarios educativos que utilizan la virtualidad como instrumento mediador facilitando el aprendizaje a distancia mediante herramientas de colaboración, interacción, y evaluación, desarrollando actividades que contribuyen no solo aprender conceptos inéditos, sino a afianzar conocimientos y habilidades, aumentando la autonomía y motivación por esta innovadora herramienta tecnológica de estudio. A través del presente artículo tipo ensayo, citando a diferentes autores se reflexiono acerca de cómo los entornos virtuales de aprendizaje representan un horizonte a transitar en la construcción del conocimiento del estudiante, se utilizó la hermenéutica como método para comprender e interpretar los aportes de los autores referenciados. Finalmente acotamos que concluyo que los entornos virtuales de aprendizaje son espacios propiciados como herramientas de actualización educativa orientados a promover el cambio en las prácticas pedagógicas tradicionales adaptándose a los nuevos retos de la educación del siglo XXI.

Palabras Claves : Conocimiento, Entorno virtual de aprendizaje, enseñanza y aprendizaje
Maiby T. Camacho $G^{*}$

Marcos E. Flores $G^{* * *}$

UPEL-IPB

Aceptado:10-09-2018

\begin{abstract}
In the globalized world technologies enable new types of learning and dissemination of knowledge, emerging various educational scenarios that use virtuality as a mediating instrument facilitating distance learning through Tools of collaboration, interaction, and evaluation, developing activities that contribute not only to learn unpublished concepts, but to strengthen knowledge and skills, increasing autonomy and motivation for this innovative tool Study technology. Through this article type essay, quoting different authors I reflect on how virtual learning environments represent a horizon to travel in the construction of student knowledge, hermeneutics was used as a method To understand and interpret the contributions of the referenced authors. Finally, I conclude that virtual learning environments are spaces conducive to educational updating tools aimed at promoting change in traditional pedagogical practices by adapting to the new challenges of 21 st century education.
\end{abstract}

Key words: Knowledge, virtual environment of learning, teaching and learnin

* Cirujano Plástico. Docente del postgrado de Cirugía Plástica Estética Reconstructiva y Maxilofacial del HCUAMP de Barquisimeto. Magister en educación superior, estudiante del Doctorado en educación convenio PIDE. Correo: maibicita@hotmail.com

* Doctorado en Gerencia, Doctorado en Cultura latinoamericana y del Caribe. Msc en Educación técnica. Docente en Educación Comercial. Tutor a todo nivel. Coordinador del eje TIC área de Currículo UPEL IPB. Correo: socram050715@gmail 


\section{LOS ENTORNOS VIRTUALES DE APRENDIZAJE: UN NUEVO CAMINO HACIA EL CONOCIMIENTO}

Maiby T. Camacho G y Marcos E. Flores G

Pp 96-109

\section{INTRODUCCIÓN}

Desde su origen la humanidad ha tenido en su esencia la necesidad tanto de descubrir como de comprender el mundo que la rodea, la educación citada históricamente como herramienta fundamental del desarrollo y la planificación del ser humano plantea la inquietud generalizada acerca de sí las experiencias educativas instauradas mundialmente serán suficientes para formar jóvenes capaces de afrontar el futuro de la humanidad.

Los conocimientos que se obtienen al terminar una profesión están cambiando; esto obliga a la actualización permanente de manera formal o informal mediante redes de aprendizaje, ambientes virtuales de aprendizaje, cursos virtuales, presenciales o semi-presenciales, promoviendo el desarrollo de un profesional altamente capacitado y acorde a las exigencias de la sociedad y el mundo globalizado

De igual manera en este mundo globalizado los datos y la información pierden valor con rapidez, considero que el conocimiento como información cambia, se reorganiza, difunde, actualiza constantemente mediante herramientas tecnológicas, por consiguiente la incorporación de entornos virtuales de aprendizaje en las actividades educativas como herramientas reales de búsqueda, selección y construcción de contenidos relevantes, de participación, e interacción con otros representa el desafío de hoy con vista al futuro.

Así pues se hace necesario que los estudiantes desarrollen las competencias tecnológicas y didácticas, que representan la dimensión pedagógica desarrollada en estos espacios tecnológicos. El ambiente virtual de aprendizaje aportara los elementos necesarios para un aprendizaje de calidad, en el que los estudiantes puedan, interactuar con sus compañeros y tutores, para adquirir información, afianzar conocimientos, como una herramienta de apoyo en el proceso de enseñanza aprendizaje, aprendiendo y reaprendiendo a lo largo de su vida.

En este orden de ideas se expusieron algunas reflexiones referidas a la utilidad de los entornos virtuales de aprendizaje recursos tecnológicos que se piensan como alternativas para complementar, diversificar o potenciar los procesos educativos, reflexivos así como 


\section{LOS ENTORNOS VIRTUALES DE APRENDIZAJE: UN NUEVO CAMINO HACIA EL CONOCIMIENTO}

\section{Maiby T. Camacho G y Marcos E. Flores G}

Pp 96-109

participativos de los estudiantes de forma autónoma, rápida, eficiente, desestructurada construyendo sus aprendizajes y aportando soluciones a interrogantes y problemáticas planteadas, mediante este mundo virtual.

Por lo anteriormente expuesto considere pertinente y oportuno reflexionar en este artículo sobre estos importantes tópicos que fueron desarrollados en concordancia con las aportes de diferentes autores cuyas perspectivas desde distintos ámbitos enriquecerán nuestra percepción del tema. A continuación sin más preludios, los invito a sumergirse en la lectura del mismo con la esperanza de poder acercarlos a un mundo apasionante: el mundo de la educación a distancia mediado por las tecnologías.

\section{EL CONOCIMIENTO: UN NUEVO HORIZONTE EN TRANSFORMACION}

El conocimiento como búsqueda de la verdad, una herramienta poderosa a través de la cual el ser humano es capaz de generar cambios, y transformaciones importantes, representa uno de los grandes temas de la filosofía de todos los tiempos, devela en qué consiste el acto de conocer, como se concibe el conocimiento, la relación entre el hombre, las cosas que lo rodean, y su origen desde los sentidos a la razón.

A pesar de que conocer es una actividad cotidiana no hay un acuerdo acerca de lo que sucede cuando conocemos algo. Platón Descartes, Aristóteles, Kant y Hume fueron algunos de los principales filósofos quienes aportaron sus teorías, como resultado de estas inquietudes surgieron corrientes filosóficas como el idealismo, racionalismo, empirismo, criticismo, todas han sido debatidas y algunas permanecen vigentes en el tiempo.

La idea más simple acerca de cómo se adquiere el conocimiento es que proviene del exterior, está en la sociedad, lo poseen los otros, nos apoderamos de él y lo incorporamos a nuestra mente. Para Habermas (1996), el conocimiento se constituye en base a intereses que han 


\section{LOS ENTORNOS VIRTUALES DE APRENDIZAJE: UN NUEVO CAMINO HACIA EL CONOCIMIENTO}

Maiby T. Camacho G y Marcos E. Flores G

Pp 96-109

ido desarrollándose a partir de necesidades naturales de la especie humana configuradas por condiciones históricas y sociales.

En referencia a esto, exponemos: todos creamos conocimientos, debido a que es una acción humana, se origina y reside en el individuo a través de un proceso cultural de intercambios sociales. Por tanto comprendo que tiene mucho que ver con la intuición, eso que está dentro de ti y te dice cómo vas a abordarlo, acá la generalización no existe porque jamás ningún ser humano será igual a otro.

Es el más polifacético e importante de todos los factores de aprendizaje tanto si puede ser medido como si no es posible determinarlo, para algunos filósofos como Kant provenía de los sentidos, mientras que para platón representaba el saber de las cosas. Reflexiono respecto a que el mismo contribuye al aprendizaje como un proceso personal, social en el cual se adquieren, transforman y aplican un conjunto de experiencias provenientes de infinitas áreas, que hace que una persona sea capaz de hacer algo que antes no era capaz de hacer, con la obtención de mejores resultados.

El proceso de aprendizaje conlleva a la producción de conocimiento, en relación con esto Nonaka (1995) distinguen dos tipos de conocimiento: el explícito que puede ser estructurado, almacenado, distribuido y el tácito: que forma parte de las experiencias de aprendizaje personales de cada individuo. En lo personal estimo que este último tipo de conocimiento simboliza la habilidad más importante para cualquier persona, grupo u organización, como herramienta de evolución.

El conocimiento es considerado una herramienta poderosa a través de la cual el ser humano es capaz de generar cambios y transformaciones importantes, se basa en la experiencia obtenida del entorno político, social, cultural, tecnológico, ecológico, y educativo, transformado por medio del lenguaje en información que gracias a los avances tecnológicos puede ser accesible a todos. Según Padrón y Bravo (2014), “el conocimiento generado a través de la información se convierte en el nuevo capital para la globalización" (p. 53), señalamos que este hecho apunta a un proceso idiosincrático y colaborativo de generación de saber. 


\section{LOS ENTORNOS VIRTUALES DE APRENDIZAJE: UN NUEVO CAMINO HACIA EL CONOCIMIENTO}

Maiby T. Camacho G y Marcos E. Flores G

Pp 96-109

Por ello la UNESCO (2010), resalta que "la sociedad dejo de ser solo mano de obra, para ser una sociedad alta en conocimiento" (p .4) centrada no solo en el proceso tecnológico, sino que se considera como un factor de cambio social. De acuerdo a este enfoque, el conocimiento será la base de las transformaciones funcionales de la sociedad esto justifica la disposición de incorporarse a los recientes cambios en los métodos educativos de enseñanza, los cuales modificaran los paradigmas tradicionales del aula y representaran novedosos retos tanto para el docente como para el estudiante, respondiendo a la necesidad de expansión tecnológica de la sociedad.

Nuestro país no escapa a este complejo proceso, inmerso en una etapa de cambios marcado por el desarrollo de entrantes avances científicos, agrícolas, tecnológicos, así como transformadores modelos educativos, currículos, contenidos programáticos, adaptados a una cambiante realidad política y social, que generara cambios en la formación y competencias del docente y el proceso de enseñanza aprendizaje, reorientando los contenidos, adaptándose a modificaciones, y avances tecnológicos del proceso de enseñanza a distancia o virtual, para la educación del siglo XXI, en una realidad que transcurre en el diario convivir, con la interacción de las prácticas cotidianas, mediadas por tecnologías.

En tal sentido Guerrero (2003) expreso que todas "las tecnologías que participan en un proceso educativo pueden considerarse, como sistemas de actuación (acción externa), pero también como fuente para la creación de innovadores modelos cognitivos o marcos de pensamiento generadores de conocimientos (representación interna)" (p. 4). Al respecto expresamos que dichos modelos conllevan al estudiante a aprender a lo largo de la vida modificando sus comportamientos y resultados, por tanto las tecnologías transformaran radicalmente la manera de gestionar el conocimiento que se aprende y reaprende.

En este escenario surge un relevante elemento clave en la construcción del conocimiento mediante herramientas tecnológicas y es la interactividad. Así al aprendizaje por información se añade al aprendizaje por interacción. La interactividad como un objeto es no obstante, solo una cara de las posibilidades de interacción: la interacción con otros alumnos, docentes o expertos abre numerosas 


\section{LOS ENTORNOS VIRTUALES DE APRENDIZAJE: UN NUEVO CAMINO HACIA EL CONOCIMIENTO}

Maiby T. Camacho G y Marcos E. Flores G

Pp 96-109

posibilidades de comunicación digital. Valdes (2015) Cito: Conforme a esto se considera que las tecnologías como herramientas interactivas representan una fuente de motivación y estímulo para aprender en los entornos virtuales debido, a la posibilidad de un mayor control sobre el propio proceso de aprendizaje, permitiendo a los estudiantes mediante el descubrimiento, la interpretación y la comunicación, construir concepto actuales, redimensionar nuevas visiones y tomar decisiones sobre ¿cómo? y ¿qué aprenden?

De acuerdo con esto cabe señalar que cuando la información es interpretada por el estudiante, la misma se transformará en conocimiento útil, al respecto el pedagogo norteamericano John Dewey durante sus estudios promovió la importancia de construir conocimientos dentro del aula a partir de la interacción y la ayuda entre pares en forma sistemática como una manera efectiva de un aprendizaje útil y valedero. Reflexiono en mi percepción, si la información tiene poco valor como elemento externo y sólo se convierte en conocimiento cuando es procesada de manera dinámica por el cerebro humano.

Respecto a este ámbito los entornos virtuales de aprendizaje tienen un papel importante en la obtención, análisis y difusión de la información resultante representando el capital intelectual del estudiante por lo cual discurro que dicho proceso representara una reciente frontera a transitar marcada mundialmente por profundos cambios educativos del siglo XXI y un nuevo reto para las futuras generaciones de estudiantes protagonistas de dichos cambios, siendo el nuevo capital del mundo globalizado.

\section{LOS ENTONOS VIRUTALES DE APRENDIZAJE, LA NUEVA PUERTA HACIA EL FUTURO DE LA ENSEÑANZA.}

La educación consiste en crear un entorno favorable, no aprendemos nada que uno mismo no haya redescubierto y reconstruido, Un aprendizaje se efectúa cuando un individuo recoge información de su entorno en función de un proyecto personal, de esta manera exponemos que un entorno virtual de aprendizaje es el espacio donde es posible gestionar el conocimiento o, 


\section{LOS ENTORNOS VIRTUALES DE APRENDIZAJE: UN NUEVO CAMINO HACIA EL CONOCIMIENTO}

Maiby T. Camacho G y Marcos E. Flores G

Pp 96-109

mejor dicho, el desconocimiento. Al respecto Salinas (2011) lo definió como: "un espacio educativo alojado en la web conformado por un conjunto de herramientas informáticas que posibilitan la interacción didáctica y el redescubrimiento"

Para Cardosi (2017) los entornos virtuales de aprendizaje juegan un papel preponderante, puesto que direccionan la manera de desarrollar procesos cognoscitivos, ofrecen herramientas para planificar y organizar la enseñanza y el aprendizaje a través de múltiples herramientas de interacción y comunicación. Desde el punto de vista del constructivismo, un entorno o ambiente sería un lugar donde los estudiantes pueden trabajar juntos y darse apoyo unos a otros así como usar una variedad de herramientas y recursos de información en la obtención de metas de aprendizaje y actividades de resolución de problemas.

Reflexionando acerca de la importancia de estilos virtuales de aprendizaje que conduzcan a adecuar a los tiempos de cambio a los futuros profesionales. Esto supone lograr una enseñanza más activa así como un mayor protagonismo de los estudiantes en su propio aprendizaje, ofreciendo grandes posibilidades de estandarización y de adecuación de los contenidos de enseñanza de acuerdo con necesidades individuales como el camino hacia un aprendizaje dinámico y flexible.

Discurro que ofrecen una serie de posibilidades para procesos de colaboración, tanto para estudiantes como tutores generando conocimientos de forma activa, formulando ideas que son compartidas, construidas a partir de reacciones y respuestas de los otros. Dicho entorno es un micro contexto conformado atendiendo a ciertas variables como lo son, en términos de Cabero (2006), el rol del profesor, el papel del alumno, la comunidad, las herramientas de comunicación, los aspectos organizativos, los contenidos, el tipo de actividades formativas, las estrategias didácticas, y los modelos de evaluación; todas estas mediadas por herramientas tecnológicas.

En ocasiones, el entorno de aprendizaje es definido como el espacio y las convenciones establecidas, pero es "la dimensión didáctica lo que convierte en un entorno de aprendizaje" Pirttiniemi y Rouvari, (s.f. pág. 7). Según el autor este entorno está organizado en cinco dimensiones: social, didáctica, física, técnica, y tecnológica, pudiendo ser estructurado en un 


\section{LOS ENTORNOS VIRTUALES DE APRENDIZAJE: UN NUEVO CAMINO HACIA EL CONOCIMIENTO}

Maiby T. Camacho G y Marcos E. Flores G

Pp 96-109

diseño tecno-pedagógico o tecno-instruccional. Proponemos que dependiendo del enfoque del autor, pueden ser vistos como un instrumento mediador, por esto surgieron las siglas EVA (entorno virtual de aprendizaje) el espacio virtual donde tendrá lugar el proceso de aprendizaje mediado por las tecnologías.

Sin embargo para Esteban (2000) quien retomo los planteamientos de Jonassen asociándolo en un modo de entender, ese proceso de enseñanza aprendizaje se concreta llevando a la práctica el diseño de las actividades, el modo de evaluar, la selección, presentación de los contenidos. Evidencio desde este enfoque un vinculó a la concepción que el docente tiene sobre el modo en que aprenderán los estudiantes, los objetivos que se propondrán y el rol que asumirá como mediador de ese proceso.

Ante estas dos visiones filosóficas del entorno virtual de aprendizaje, pienso que podemos interrelacionarlas puesto que evidentemente es el espacio en el que ocurrirá el proceso de aprendizaje que en los sistemas educativos está en las aulas, también podemos visualizar y reflexionar acerca de cómo ocurre este proceso, que elementos son necesarios, de qué manera podrían ser mejorados, y cuál es nuestro papel en el proceso.

En relación a lo anteriormente señalado, surgió un concepto innovador descrito por Onrubria (2017), quien habla de triangulo interactivo, que se establece entre la persona que aprende (Alumno), la persona que enseña (Profesor) y el contenido que se pretende enseñar y aprender(Contenido), estas herramientas actuaran mediando la actividad conjunta entre estas variables hasta constituirse en configuradoras de un entorno, espacio de trabajo y aprendizaje bien sea para la formación, o para la actualización.

Los entornos virtuales de aprendizaje como estructura organizativa particular del sistema educativo aportan competencias necesarias para la adaptación de los estudiantes al aprendizaje permanente que podrían ser hoy construidas en ausencia de la virtualidad, al respecto Henao (2005) describe: "los ambientes virtuales de aprendizaje ofrecen un potencial enorme para mejorar la calidad de la evaluación académica tanto en la educación presencial como a 


\section{LOS ENTORNOS VIRTUALES DE APRENDIZAJE: UN NUEVO CAMINO HACIA EL CONOCIMIENTO}

Maiby T. Camacho G y Marcos E. Flores G

Pp 96-109

distancia". (p. 37). Por cuanto expongo que estas herramientas tecnológicas transforman las crecientes políticas educativas del mundo.

La inclusión tecnológica se ha instalado definitivamente en la concepción educativa colectiva como una necesidad y un logro social a alcanzar se ha asociado básicamente a resolver las necesidades de sociedad que demanda una educación permanente, ampliando la cobertura para el acceso a la educación, ofreciendo lo que hoy en día denominan la democratización del acceso a la educación, en el establecimiento de esta sociedad de aprendizaje permanente se propicia el surgimiento de sociedades del conocimiento pluralistas y participativas que sepan integrar en vez de excluir, tal como lo expresa la UNESCO (ob. cit.):

....garantizar una educación inclusiva y equitativa de calidad y promover oportunidades de aprendizaje permanente para todos, esto representa una empresa colectiva en la que todos los participantes hacen un esfuerzo concertado para cumplir con sus responsabilidades (p. 5)

Mencionamos que esto representa una tarea colectiva en la que todos los participantes hacen un esfuerzo concertado para cumplir con sus responsabilidades educativas, siendo los Entornos Virtuales de Aprendizaje (EVA), la herramienta más idónea para este proceso transformador y generador en el desarrollo del sistema curricular educativo. Según Velásquez (2007), es imposible obviar el auge y crecimiento que actualmente se observa en la educación a distancia, especialmente en las principales instituciones de educación superior, como una alternativa de brindar una educación flexible y de calidad a las grandes masas de la población, basada en métodos educativos innovadores, en el cual las nuevas tecnologías, tiene gran un impacto.

La educación virtual da la oportunidad al estudiante, de elegir sus horarios de estudio, por otra parte, es una excelente herramienta que puede ayudar a los usuarios no sólo a aprender conceptos novedosos sino también a afianzar conocimientos y habilidades, aumentado así la autonomía y la motivación de los estudiantes por diferentes temas. En tal sentido Onrubia (ob. cit.) expresa que: 


\section{LOS ENTORNOS VIRTUALES DE APRENDIZAJE: UN NUEVO CAMINO HACIA EL CONOCIMIENTO}

Maiby T. Camacho G y Marcos E. Flores G

Pp 96-109

Las prácticas académicas que utilicen entornos virtuales de aprendizaje (EVA), supone retos abordables para el alumno; en el sentido de que pueda resolverlos o solventarlos por sí solo, sino de que pueda afrontarlos gracias a la combinación de los instrumentos y de sus propias posibilidades motivados por aprender y mejorar su rendimiento educativo (p.17).

Es así como el correcto funcionamiento de un (EVA) facilita tanto la interacción, como la construcción de conocimiento en forma colaborativa al interior de una comunidad de aprendizaje proporciona al proceso de enseñanza y aprendizaje, herramientas útiles, sin necesidad que se produzca un encuentro presencial entre estudiante, docente, ni en el espacio ni en el tiempo, asumiendo las funciones de contexto de aprendizaje, que el aula desarrolla en el entorno presencial.

Bajo esta perspectiva, las actividades cooperativas, desde el área de la tecnología, son ejes de innovación en aspectos socio-culturales propios del entorno pedagógico, por cuanto desarrollan en los estudiantes capacidades meta cognitivas que le permiten continuar aprendiendo toda la vida. Igualmente, garantizan la interacción y la comunicación en lugares distantes para compartir la información sin fronteras geográficas. Por consiguiente, este tipo de actividad rompe con la enseñanza unidireccional pasiva, mejora la autonomía de quien aprende, para que actúe con libertad, descubra las cosas por sí mismo y construya su aprendizaje.

En torno a esto reparo que los estudiantes al construir novedosos conocimientos, encuentran la motivación para la aplicación de los mismos en ideas, que frecuentemente se transforman en proyectos. Poseen conciencia de la importancia de la tecnología como requisito de cualquier proyecto para construir el propio esquema de vida. Se destacó la constancia como herramienta indispensable para la obtención de logros significativos, que permitan construir su propia proyección personal, aporte invaluable a la comunidad donde se desenvuelva.

Finalmente señalo los entornos virtuales de aprendizaje como un ambiente abundante de datos e información para la construcción de aprendizaje cotidiano, permitiendo a quienes acceden a este recursos, la posibilidad de desarrollar, seleccionar, validar y construir información, de utilizar esta información como insumo para la construcción de innovadores 


\section{LOS ENTORNOS VIRTUALES DE APRENDIZAJE: UN NUEVO CAMINO HACIA EL CONOCIMIENTO}

\section{Maiby T. Camacho G y Marcos E. Flores G}

Pp 96-109

conocimientos, representando la aplicación de aprendizajes para el desarrollo de las acciones como agente de cambio.

Debemos comprender que esto solo representa un elemento que sin el uso del estudiante no podrá generar un cambio por tanto como protagonista de este le corresponde la responsabilidad de utilizarlo adecuadamente.

\section{CONCLUSIONES}

Una vez expuesto las diferentes enfoques sobre los entornos virtuales de aprendizaje considero que dichos entornos favorecen conductas, actitudes, conocimientos y sentimientos variados en cada uno de los estudiantes, estos pueden aprender distintos tópicos y al mismo tiempo nutrirse de estos ambientes tecnológicos, por consiguiente tendríamos que organizar los contextos y los ambientes de aprendizaje para que los estudiantes se formen promoviendo situaciones más promisorias, variadas, sugerentes y permisivas en la cual cada uno de ellos como individuos encuentren en la diversidad suficientes elementos para plasmar su personalidad, ajustar sus capacidades y conocimientos e integrarse a la comunidad.

Este método de enseñanza virtual, perfilado como uno de los muchos recursos que permiten ofrecer al estudiante la posibilidad de asimilar conceptos, desarrollar habilidades y resolver problemas de diversa complejidad, facilita la comprensión y desarrollo de competencias, destrezas y métodos sistemáticos para el logro de los aprendizajes significativos, afianzando rasgos de comunicación, cooperación e interacción social que representaran un novedoso modelo de aprendizaje lineal, interactivo, divertido, desafiante basado en la construcción y el descubrimiento del saber, personalizado, centrado en la persona que aprende quien decide ¿Qué?, ¿Cuándo¿ y ¿Cómo¿ y ¿de qué manera?.

Nuestros esfuerzos deberán centrarse en acompañar al estudiante durante este proceso, así como a los entes gubernamentales y las políticas educativas le corresponderían estrechar la brecha digital permitiendo un mayor acceso a estas herramientas tecnológicas. 


\section{LOS ENTORNOS VIRTUALES DE APRENDIZAJE: UN NUEVO CAMINO HACIA EL CONOCIMIENTO}

Maiby T. Camacho G y Marcos E. Flores G

Pp 96-109

Me planteo la inquietud de que el conocimiento no es estático entonces: ¿cómo suponer que sabemos algo de los cambios que nos esperan dentro de dos décadas? por tanto los entornos virtuales de aprendizaje, representan el escenario para el desarrollo de competencias tecnológicas dentro de una vasta clasificación de modalidades educativas interactivas, didácticas, cognitivas que permiten al estudiante al docente transitar en este camino nunca acabado hacia la adquisición del conocimiento, teniendo en cuenta que no debe perderse de vista que el aprendizaje es lo relevante, siendo fundamental una visión multidisciplinar que permita una transformación de la educación a distancia, el proceso de enseñanza aprendizaje acorde a innovaciones, avances tecnológicos existentes y cambiantes en el mundo.

\section{REFERENCIAS}

Cabero (2006) Cabero, J. y P. Román (2006) E-actividades. Un referente básico para la formación en Internet. Eduforma. Sevilla.

Cardosi, E (2017) Gestión del conocimiento basada en entornos personales de aprendizaje desde la comunidad digital. Tesis doctoral sin publicar. Universidad Yacambu. Barquisimeto edo. Lara Agosto 2017

Esteban, M (2000) El diseño de entornos de aprendizaje constructivista. En Revista de Educación a Distancia. Murcia. Número 8. Edición electrónica :http://www.um.es/ead/red/6/documento6.pdf [Consultado: 2017, noviembre 05]

Guerrero, C. (2003) Los entornos virtuales de aprendizaje como Instrumentos de mediación. [Versión electrónica]. Teoría de la educación: educación Y cultura en la sociedad de la información.Vol 4. Ed. Univ. de Salamanca. Consultado el 07/11/17 en: http://campus.usal.es/ teoriaeducacion/rev_numero_04/n4_art_suarez.htm

Habermas, J. (1996). La teoría de la acción comunitaria. Madrid: Cátedra.

Henao, O. (2005). La evaluación en un entorno virtual. [Documento en línea]. Disponible en: http://www.colegiovirtual.org/pr03_14.html. [Consulta: 2017, Noviembre 03] .

Nonaka, I (1995) The knowledge creating company 


\section{LOS ENTORNOS VIRTUALES DE APRENDIZAJE: UN NUEVO CAMINO HACIA EL CONOCIMIENTO}

\section{Maiby T. Camacho G y Marcos E. Flores G}

Pp 96-109

Onrubia, J. (2007) Aprender y enseñar en entornos virtuales: actividad conjunta, ayuda pedagógica y construcción del conocimiento (documentos) RED: Revista de Educación a Distancia, ISSN 1578-7680, núm. 2, 2008.

Padrón, C y Bravo, M. (2014).Competencias tic para la gestión del conocimiento: un aporte desde el modelo Tpack. Revista EDUCARE, v.18, n. 3, p.49, Dic. 2014. Disponible en: http://revistas.upel.edu.ve/index.php/educare/article/view /360/168. [Consultado: 2016, septiembre 13]

Pirttiniemi y Rouvari, s.f.) Pirttiniemi, E. y Rouvari, A. (s.f) Dimensión didáctica del Entorno de aprendizaje. Consultado el 4/11/17 en: http://www.cibernarium.tamk.fi/havainnollistaminen_es/didactic_environment.htm

Salinas, I (2011). Entornos Virtuales de Aprendizaje en la Escuela: tipos, modelo didáctico y rol del docente. Pontificia Universidad Católica Argentina (UCA). Disponible: http://www.uca.edu.ar. [Consultado: 2018, julio 23]

UNESCO. (2010), "Fundamentos y marco conceptual para las (TIC) y la formación docente", las tecnologías de la información y comunicación en la formación docente, [en línea], disponible en: http://www.dhl.hegoa.ehu.es/recursos/636.[Consulta: 2016, Septiembre 20]

Valdes R, Maria Caridad (2015) "La interactividad en escenarios pedagógicos virtuales universitarios" Ponencia Eduqa 2015. [Documento en línea] disponible en http://www.eduqa.net/eduqa2015/images/ponencias/eje5/5_am_VALDES_Maria_La_interactividad_e s_escenarios_pedagogicos_virtuales_universitarios.pdf

Velásquez, K. (2007).Universidades venezolanas incursionan en la educación a distancia. Especiales Universia. [Documento en línea]. Disponible:http://servicios.universia.edu.ve/rediseno/contenidos/tu/tu.php?seccion=transform acion\&reportaje=11359. [Consulta: 2016, Agosto 14] 